\title{
La regulación del comercio en Bolivia: de la economía informal al mercado extralegal
}

\section{Trade regulation in Bolivia: from informal economy to extralegal market}

Juliane Müller*

\section{Resumen}

Este artículo estudia el mercado de equipos electrónicos en La Paz. Se analizan los mecanismos sociales y los discursos alrededor de la regulación de la competencia y de la cooperación entre los diferentes actores, negocios familiares y empresas multinacionales. Se busca mostrar que el mercado está constituido por diversos espacios de regulación cuyos actores co-producen y aplican normas no necesariamente codificadas por el sistema jurídico. Se propone la noción de "mercado extralegal" para conceptualizar estos espacios que hacen referencia a fuentes sociales y empresariales de autoridad y legitimidad para el control de la competencia y la coordinación de las transacciones.

Palabras clave: Sociología económica; Antropología económica; Economía cultural; Costumbres; Normas sociales.

\section{Abstract}

This article studies the market for electronic devices in La Paz. It analyzes social mechanisms and discourses around the regulation of competition and the cooperation between different actors, family businesses and multinational enterprises. The paper aims to show that the

* Doctora en antropología, investigadora y docente de la Universidad de Múnich (LMU), Alemania.

Contacto: juliane.mueller@ethnologie.Imu.de 
market is constituted by diverse spaces of regulations and actors who co-produce and apply norms not necessarily codified by law. The notion of "extralegal market" is proposed to conceptually approach these spaces that refer to social and entrepreneurial forms of authority and legitimacy in order to control competition and coordinate transactions.

Keywords: Economic Sociology, Economic Anthropology, Cultural Economics, Customs, Social Norms.

Clasificación/Classification JEL: Z13, Z10, A13.

\section{Introducción}

Dos categorías predominan en el análisis del comercio en Bolivia y en América Latina, lo informal y lo popular. Mientras que la primera categoría abarca estudios sobre desarrollo dependiente, neoliberalismo y comercio urbano y en vía pública (CEDLA, 1986; FernándezKelly y Shefner, 2008; Pereira, 2009; Portes y Schauffler, 1993; Rojas, 1992), la segunda se concentra en el análisis del tramado de redes socio-comerciales, enfocando agentes nativos y lógicas propias (Matos Mar, 1984; Quijano, 1998; Tassi et al., 2013; Tassi, Hinojosa y Canaviri, 2015). Los temas político-legales son importantes en el análisis del comercio informal, ya que éste se define principalmente por comercializar productos y servicios lícitos pero fuera de la supervisión del Estado ${ }^{1}$. Los estudios sobre economía popular, a su vez, se centran en las instituciones y redes "debajo del radar de la ley" (Mathews y Yang, 2012).

Este artículo se basa en el corpus de estudios sobre economía informal y popular. El enfoque, sin embargo, es algo distinto. Se considera que el comercio popular no puede ser definido como fuera de la ley, ya que los comerciantes se mueven en un espacio legal ambiguo. Su formalización a medias y el reconocimiento estatal de sus asociaciones, así como la constante negociación entre gremiales, funcionarios y empleados de grandes empresas, cuestionan la idea de fronteras nítidas entre lo formal y lo informal, lo legal y lo ilegal. Desde la sociología económica y la antropología de los mercados, además, el comercio popular no es únicamente un objeto de estudio en sí mismo, sino forma parte del funcionamiento de mercados más amplios.

1 A diferencia de los "mercados ilegales", donde los objetos intercambiados, el intercambio mismo o las condiciones laborales de producción son ilegales y/o ilícitas, en el comercio informal los productos en sí suelen ser legales y socialmente lícitos, pero el intercambio mercantil sale de regulaciones oficiales, como la tributaria y la propiedad privada e intelectual. 
Mientras que los trabajos sobre economía popular centran su mirada en las zonas fuera de "las normas de las autoridades formales" (Lins Ribero, 2012:44; véase Rojas, 2016), explicando la generación y reproducción de estos espacios principalmente desde las historias, identidades e institucionalidades en el interior de la economía popular (Tassi et al., 2013), el presente texto aborda la producción de diferentes espacios de regulación a partir del encuentro de una serie de agentes con disposiciones y experiencias distintas. Este cambio de perspectiva implica dos objetos claves para el artículo: por un lado, el análisis de las relaciones de cooperación y competencia entre agentes diferentemente posicionados en un mercado parcialmente fuera de la ley y, por otro lado, el estudio de las negociaciones y disputas acerca de lo legal y lo legitimo entre los actores involucrados. Este enfoque da cuenta de la heterogeneidad de la economía boliviana sin predeterminar esferas separadas y lógicas opuestas (Wanderley, 2012). La economía popular forma parte de una red de relaciones sociales tanto horizontales y con potencial para convertirse en una "economía de base ancha"2, como también verticales hacia empresas más grandes y empresas multinacionales.

Se analiza un mercado que nace a partir del extraordinario dinamismo del comercio popular, pero que se extiende más allá de los circuitos comerciales del mismo. Se parte de una definición básica de mercado como espacio de intercambio voluntario regular de bienes y servicios con base a precio bajo condiciones de competencia (Aspers y Beckert, 2008). Un mercado es establecido por actores interesados en participar del mismo ya sea como oferentes o demandantes.

El mercado de artefactos electrónicos es especialmente tangible en ciertos espacios físicos, como las calles Eloy Salmon y Huyustus en La Paz, el Gallo en Cochabamba y la calle Isabel la Católica en Santa Cruz, pero también se extiende más allá de estos locales concretos. El mercado es un campo social relacional formado históricamente y se constituye por la interdependencia entre los agentes que se observan mutuamente y actúan en relación los unos con los otros (White, 2002), dentro de una estructura histórico-política de relaciones de fuerza (Bourdieu, 2001:25).

2 La "economía de base ancha" es definida por la co-existencia de micro, pequeñas y medianas empresas productivas en ciertos sectores y lugares específicos (Gray Molina, 2007; PNUD, 2005). Por el énfasis que pone en el desarrollo industrial a partir del tejido social de pequeñas unidades productivas y empresas familiares, se asemeja a la noción del cluster (Porter, 2000; para América Latina, véase Felzensztein et al., 2014; Giuliani, Pietrobelli y Rabellotti, 2005). El comercio informal no suele ser mencionado como un potencial para la formación de clústeres locales, como máximo aparece como "survival cluster" con pocas posibilidades de persistir (Altenburg y MeyerStamer, 1999). Para una lectura crítica del concepto de clúster en relación con el comercio popular, véase Müller (2017). 
Esta noción de mercado distingue la economía de mercado de la economía de libre mercado o neoliberal (Wanderley, 2012), distinción importante que rechaza la visión ahistórica de que economías de mercado destruyen necesariamente instituciones locales y relaciones de cohesión y solidaridad. El modelo de "mercados multiples" (Zelizer, 2011) postula que mercados empíricos no se adecuan al modelo ortodoxo neo-clásico de lógicas exclusivamente económicas y maximadoras. Y va más allá al afirmar que ningún mercado existe fuera de sus estructuras particulares, las cuales están imbricadas en circunstancias históricas y culturas particulares (Dilley, 1992).

Pese a que el Estado ejerce un fuerte rol normalizador y homogeneizador sobre el funcionamiento de los mercados y la acción de sus participantes, la regulación económica y financiera oficial no necesariamente es cumplida a cabalidad en muchos países de la región y de otros continentes como en África occidental (Guyer, 2004). En Bolivia, el Estado protege derechos y mecanismos imprescindibles para el funcionamiento regular del intercambio mercantil (seguridad ciudadana, propiedad privada, establidad monetaria), pero no penetra todos los espacios, y en muchos rubros comerciales no regula todas las áreas (propiedad intelectual, estándares de calidad, regulación de la competencia). De esta manera la regulación también es ejercida por los diversos agentes privados que crean "fuentes sociales de autoridad" con "reglas vinculantes basadas en sanciones" (Smart y Zerilli, 2014:225; traducción hecha por la autora).

Recientemente nuevos estudios en sociología económica han ampliado el conocimiento sobre "mercados ilegales", ya que van más allá de los análisis sobre el comercio de bienes ilícitos y flujos transfronterizos (Van Schendel y Abrahams, 2005; Blanes, 2017), prestando atención a la complejidad de los procesos económico-legales detrás del orden social de los mercados (Beckert y Wehinger, 2011; Dewey, 2016; Beckert y Dewey, 2017). Al superar el supuesto de separación nítida entre lo legal y lo ilegal con la noción de lo extralegal, la cual complejiza las fronteras entre ambas esferas, el presente estudio etnográfico pretende aportar insumos a la investigación sobre los mercados que funcionan al margen del sistema jurídico oficial.

Este artículo busca esbozar las características de un tipo de mercado que se puede clasificar como "extralegal", siendo extralegality un concepto que hace referencia a áreas no propiamente definidas por la ley y a formas de autoridad y normas sociales no codificadas por el derecho estatal. Desde el punto de vista antropológico, y en un contexto como el boliviano, es importante desvincular la asociación entre mercados extralegales y formas de gobernanza de grupos criminales como las mafias; asociación que ha predominado en los enfoques desde las 
ciencias políticas y en menor medida desde la sociología (Beckert y Dewey, 2017). En cambio, los estudios desde la antropología se han enfocado en movimientos sociales y territorios indígenas, los cuales desafían las jerarquías inscritas en ciertos reglamentos oficiales y pueden llegar a construir "legalidades alternativas" (Smart y Zerilli, 2014:225). Esta perspectiva ofrece ventajas para analizar un mercado específico, en este caso de artefactos electrónicos y estudiar cómo los productos cambian su estatus legal en función de los espacios de regulación por los cuales atraviesan. En palabras de Van Schenderl y Abrahams (2005):

... zonas donde una serie de normas o reglas son dominantes, y éstas pueden ser generadas desde el Estado o producidas socialmente. Los regímenes regulatorios organizan rutinas, hacen respetar reglas, permiten o restringen acceso a recursos, definen o mantienen fronteras, identifican yexcluyen actores (...) Los espacios regulatorios son siempre disputadosybordeados por umbrales de confianza y de violencia (pp.15-16; traducción hecha por la autora).

El documento se concentra en la problemática sociológica de cómo entender la regulación de la competencia y la cooperación en un mercado parcialmente fuera de la ley, con base en el estudio del mercado de equipos electrónicos en La Paz. El acápite 2 analiza las normas y estrategias de regulación de la competencia al margen del orden estatal-jurídico y las justificaciones de los comerciantes para sus acciones. En el acápite 3 el foco de atención se dirige a las relaciones entre comerciantes y empresas transnacionales en las dinámicas económicas extralegales. En el centro del análisis está la disputa por los significados de los términos del derecho comercial entre comerciantes, empresas transnacionales y actores estatales. El documento concluye con una reflexión sobre el concepto de mercado extralegal.

El estudio adoptó una estrategia metodológica con base en entrevistas semi-estructuradas, observaciones etnográficas y conversaciones prolongadas con comerciantes y empleados de las empresas transnacionales en sus lugares de trabajo. El seguimiento meticuloso de diferentes mercados de artefactos y de las redes comerciales transnacionales ocurrió entre 2013 y 2017. También se realizó un análisis sistemático de notas de prensa y entrevistas a ejecutivos empresariales, funcionarios públicos, abogados y las casas comerciales en la zona franca de Iquique (Chile) $)^{3}$.

3 A lo largo del texto, todas las citas directas de mis interlocutores son documentadas con el lugar y la fecha de la entrevista o conversación. 


\section{El mercado de artefactos electrónicos en La Paz y los espacios de regulación en su interior}

La sociología económica estudia empíricamente cómo las redes sociales, instituciones y convenciones llegan a regular la competencia y garantizar la cooperación en los mercados. Se evidencia la importancia de las dinámicas sociales para la estabilización de las transacciones y la reducción de la incertidumbre, especialmente en mercados heterodoxos que no cumplen a cabalidad el marco legal estatal (Beckert, 2007; Beckert y Wehinger, 2011). Complementariamente, la antropología ha estudiado los mercados basados en un entramado complejo de relaciones socio-comerciales e instituciones no reguladas por el sistema burocrático-jurídico (entre otros, Geertz 1978; Clark, 1994). Estos mercados también son contextos discursivos y morales en que la legalidad y la legitimidad de las acciones están en constante disputa. En este sentido, el estudio del uso de los términos del derecho comercial por parte de los actores para justificar sus comportamientos es muy importante para entender la regulación de la competencia en un mercado extralegal.

\subsection{La regulación de la competencia}

Para ser rentables y minimizar la incertidumbre, los proveedores y otros agentes de un mercado buscan garantizar la competencia de precios. En los mercados legales, los grados de monopolización, oligopolización y la formación de prácticas anti-competitivas y de barreras de entrada al mercado son determinados tanto por la capacidad y voluntad del Estado para regular como también por la relación de poder entre los agentes económicos. En estos mercados, el Estado juega un rol primordial a través del derecho de la competencia, de la propiedad privada e intelectual (Beckert y Wehinger, 2011:7; Fligstein, 1996).

En los mercados extralegales, los agentes económicos no utilizan únicamente o primordialmente las normas y los mecanismos jurídico-estatales para regular la competencia. Cómo veremos a continuación, los comerciantes de artefactos de la ciudad de La Paz, organizados en asociaciones gremiales, establecen normas y estrategias propias para controlar la entrada de agentes ajenos y las justifican como prácticas anti-monopolizadoras.

Hace algunos años, una de las empresas transnacionales coreanas de electrónica presente en Bolivia intentó disminuir el número de los comerciantes de sus productos para trabajar exclusivamente con un solo cliente distribuidor, el cual sería una empresa de línea blanca originaria de la ciudad de Santa Cruz con sede en la calle Eloy Salmón de la ciudad de La 
Paz. Los comerciantes de artefactos de La Paz reaccionaron con un boicot a la marca y se manifestaron enfrente de su sede en Santa Cruz hasta que ésta dio marcha atrás.

Otro caso ocurrió con una distinta transnacional coreana que buscó instalar un servicio de entrega exclusivo para sus productos originales con garantía de la marca. En esta oportunidad la reacción vino de chóferes y transportistas. Un tercer caso, también reciente, se dio cuando la asociación que aglutina tiendas y puestos de un rubro electrónico de la Huyustus y Calatayud expresó a comisionarios de casas comerciales de Iquique que éstos no podían "apropiarse del mercado boliviano como suyo" (La Paz, 13.11.2015). Esta "falta de respeto" ha sido reclamada por el directorio de la asociación con "autoridad", lo que implicó manifestaciones por parte de una colectividad numerosa, acusaciones y amenazas verbales. Estas acciones colectivas han obligado a las marcas Epson, Samsung, HP, LG y otras a "trabajar con nosotros", y condujeron a acuerdos orales prohibiendo la venta libre a otras tiendas y supermercados en la ciudad de La Paz según el directorio de la asociación. (La Paz, 13.11.2015).

Pese a que no todas las asociaciones de artefactos en el área Eloy Salmón, Buenos Aires, Huyustus y Calatayud tienen capacidad de protección tan fuerte de su mercado, de una forma u otra ellas actúan a favor de los intereses de sus afiliados, los comerciantes importadores, mayoristas y minoristas, frente a las medianas empresas nacionales y de Iquique, y las grandes transnacionales. Las justificativas por parte de los comerciantes para sus acciones de control de la entrada de nuevos agentes económicos son la defensa de "su mercado" ante terceros que representan una "competencia desleal" al no "respetar" el mercado y la asociación a cargo de supervisarlo.

El hecho de que el término "competencia desleal" se repita muchas veces en estos contextos es significativo. El Código Penal se refiere a lo desleal en el artículo 237 sobre el "Desvío de clientela"; dice "valiéndose de falsas afirmaciones, sospechas, artilugios fraudulentos o cualquier otro medio de propaganda desleal, (...) en beneficio propio o de un tercero y en detrimento del competidor, para obtener ventaja indebida, (...)". El Código de Comercio define en el capítulo V (artículos 66 a 71) una serie de actos de competencia desleal que apuntan en la misma dirección: competencia desleal según el marco legal en Bolivia refiere a confundir al cliente en cuanto al origen y la calidad del producto y sacar provecho de eso frente a competidores.

Competencia desleal es un término moral que implica fraude y malas intenciones. El uso de este término por los comerciantes insinúa justamente eso: que competidores nacionales 
y extranjeros no tienen la legitimidad moral de interferir en su mercado y no juegan limpio al intentar hacerlo. Sin embargo, según las normas oficiales, las acciones de los comerciantes gremiales, descritas anteriormente, no son prácticas en contra de competencia desleal.Más bien podrían ser consideradas acciones anti-monopolizadoras (en contra de un solo distribuidor) y acciones proteccionistas (barreras a la entrada que desincentivan la competencia). En ambos casos estas prácticas informales están cumpliendo un rol de supervisión de la competencia.

En este mercado, las disputas entre los agentes económicos son solucionadas por la vía de acuerdos verbales y no a través del sistema jurídico, pese a que el primer caso podría ser denunciado ante la Autorización de Fiscalización de Empresas (AEMP), dependiente del Ministerio de Desarrollo Productivo y Economía Plural, que actúa en casos de conductas anticompetitivas ${ }^{4}$, aunque la normativa anti-competitiva no se encuentra muy desarrollada en Bolivia5. También la NCPE (artículo 314) y el Código Penal (artículo 233) prohíben el monopolio privado de importación, producción y distribución de mercadería. Ante esa normativa y desde el punto de vista de la AEMP, cualquier persona natural o jurídica (por ejemplo, gremios de comerciantes con personalidad jurídica) podría denunciar un delito de conducta anti-competitiva que sería interpretado como un delito de orden público (La Paz, 18.09.2017).

Lo interesante de estos casos está en la elección de los comerciantes de no acudir a la AEMP. Más bien prefieren aplicar sus propias normas con respecto a las fronteras de su mercado, y la inclusión y exclusión de actores, y también adoptar estrategias colectivas para su cumplimiento. En otras palabras, a través de estas acciones ellos construyen un espacio de regulación al margen del estatal-jurídico.

\subsection{La regulación de los productos, cooperación y confianza}

Como vimos en el acápite anterior, la competencia desleal implica, desde el marco jurídico, infracción de marcas, lo que ocurre diariamente en los mercados populares de La Paz y Bolivia. De hecho, la comercialización de falsificaciones es omnipresente en muchas regiones del mundo, incrustada en circuitos comerciales transfronterizos (Van Schendel y Abrahams, 2005). Muchas veces la venta final de estos productos es efectuada en mercados que son legales. En estos casos, el intercambio de imitaciones forma un nicho dentro de un mercado

4 Capítulo III de la Ley 29519 de 2008, que las prohibe.

5 No existe una institución que aglutina la regulación de la competencia en todos los sectores y mercados. Un nuevo Código de Comercio más desarrollado está pendiente desde hace años (La Paz, 24.08.2017). 
más amplio (ej. La feria La Salada en Buenos Aires, mercados de distribución al por mayor en China y los mercados de artefactos electrónicos en La Paz).

En estos mercados, la propiedad privada suele ser protegida en tanto no son espacios extraterritoriales donde la policía no entra y la justicia no aplica. Además existe una institucionalidad, basada en el control de las redes sociales, que garantiza el intercambio regular de los productos, al mismo tiempo que éstos suelen salir del marco legal nacional e internacional sobre propiedad intelectual. En el mercado de celulares en Bolivia, por ejemplo, existe una escala graduada de categorias de equipos desde un original nuevo con garantía oficial, un original nuevo sin garantía para Bolivia, una imitación nueva, un producto de segunda mano original (en ocasiones robado), uno de segunda mano "refurbished" ${ }^{\text {y hasta }}$ una imitación de segunda mano.

Por la notoria transgresión de las normas oficiales en Bolivia, se podría esperar que las transnacionales apelen a instancias jurídicas para impedir la infracción de las marcas, como ocurre en otros países de América Latina (Dent, 2012; Pinheiro-Machado, 2013). Sin embargo, según las mismas empresas y el Servicio Nacional de Propiedad Intelectual, son pocas las demandas empresariales sobre infracción de propiedad intelectual en el país. Las que hubo fueron en el rubro de ropa y zapatos. Se conoce un reclamo en 2013 de una compañía brasileña de línea blanca (La Paz, 27.10.2016). En diciembre de 2015, la policía desarticuló dos talleres acusados de imprimir stickers falsos para electrodomésticos (La Razón 24.12.2015). En lugar de las demandas legales, las cuales tienen baja probabilidad de éxito y alta de "pérdida de recursos" (La Paz, 26.05.17), las transnacionales optan por presionar a las asociaciones de comerciantes para que controlen la circulación de falsos stickers, como los de LG Electronics hace algunos años.

La manera más sistemática y exitosa de las transnacionales para ingresar al mercado de artefactos en las grandes ciudades bolivianas ha sido dejar que se vendan productos sin garantía o imitaciones, y al mismo tiempo incentivar fuertemente la venta de los productos originales con garantía para Bolivia. Las marcas dan bonos, premios y combos a los vendedores y dueños de negocios de la Eloy Salmón, Buenos Aires y Huyustus a cada producto original con garantía vendido (en el caso de los celulares, éstos son los homologados).

6 "Refurbished" refiere a productos electrónicos previamente devueltos al productor o comercializador. A diferencia de los productos usados de segunda mano, suelen ser reparados y re-ensamblados antes de ser revendidos. 
Las transnacionales también apoyan a los locales más leales con publicidad pagada y otros materiales. Las empresas conocen la gran importancia de los vendedores en los mercados, pues son éstos quienes tienen el contacto directo con los clientes e influyen en la decisión de ellos por una u otra marca. Las empresas capitalizan este poder de persuasión en el contacto cara-a-cara y también la práctica, bien arraigada en el mercado, de complementacion del sueldo de los vendedores por producto vendido. Las marcas potencian ese sueldo a través del pago de bonos por la venta de productos originales con garantía oficial. De esa manera incentivan la venta de estos productos originales.

Aunque siga existiendo mucha venta fuera del control de las marcas, éstas han logrado subir la venta de los productos originales con garantía oficial de manera significativa en los últimos años. Es más, la creación de esa nueva categoría de productos les sirve a las empresas de manera extraordinaria para empezar a controlar la logística, hacer seguimiento post-venta y generar fidelidad en el cliente. Después de la compra, los clientes finales registran los productos con la empresa (para tener acceso a ciertas aplicaciones y servicios extras) y así permiten que cada mercadería registrada sea seguida desde la fábrica hasta los mercados en Bolivia. También facilitan el manejo de los datos personales de sus clientes, el cual es un elemento clave para el marketing en línea.

La acumulación de información digital sobre la cadena de acopio y los clientes finales evoca la ilusión de hacer legible y legal al mercado popular. Es, sin embargo, una legalidad empresarial que no coincide con la legalidad estatal, ya que todas las mercancías, originales con garantía o truchas, entran al país de la misma manera, mayoritariamente por rutas de contrabando. También es importante notar que las empresas transnacionales no cambian las costumbres transaccionales en los mercados (regateo de los precios, venta sin factura y pago de IVA) y no prohiben la venta de productos falsos o de segunda mano, sino incentivan la venta de los productos originales. De la misma manera, no homogeneizan la oferta que sigue siendo variada, con celulares en una escala de por lo menos seis registros. Por ende, al imponer sus reglas propias acerca del reconocimiento de los productos, las transnacionales están creando su propio espacio de regulación, el cual no coincide con el definido por el marco estatal-jurídico. Para tal fin ha sido imprescindible crear relaciones duraderas de confianza con los comerciantes.

La confianza es un recurso esencial en cualquier mercado. Comprobar si uno puede confiar en la contraparte comercial es un asunto más urgente en mercados no propiamente supervisados por el aparato estatal. La literatura sociológica distingue dos tipos de confianza: 
confianza en relaciones personales y confianza en instituciones (Giddens, 1990). En el comercio popular donde reina la confianza en relaciones y personas más que en instituciones oficiales (entre otros, véase Tassi et al., 2013), uno tiene que dar signos de honradez y construir una buena reputación a largo plazo. La persona en su totalidad tiende a ser evaluada, no solamente en su rol comercial. Dicho eso, podemos constatar que muchas veces las relaciones personales en la economía popular son contrapuestas analíticamente a las relaciones supuestamente siempre abstractas e impersonales en el mundo de la gran empresa. En el caso del mercado de artefactos, vemos que las empresas transnacionales están fuertemente involucradas en el tejido social local y las relaciones personalistas e informales.

Las empresas transnacionales más activas en los mercados de equipos electrónicos en La Paz y Bolivia son las del este asiático. Mientras que uno encuentra mercancías de empresas euro-americanas (por ejemplo, Philips, Oster, Bosch), predominan productos asiáticos. Transnacionales japonesas, coreanas y chinas son las únicas con una presencia directa en los mercados populares ${ }^{7}$. Las mismas tienen empleados exclusivamente en estos mercados, quienes diariamente realizan visitas a los puestos y tiendas para incentivar y supervisar la venta de productos originales. Una de las empresas coreanas tiene asignados 29 empleados solamente para el área Eloy Salmón, Buenos Aires, Huyustus y Calatayud (entre asistentes de venta, merchandisers, promotores y supervisores).

Desde que trabajan en el corazón del comercio popular, las marcas han ido madurando. Han aprendido cómo manejar estos mercados y cómo relacionarse con sus diversos clientes comerciales. Buena parte de los procesos de aprendizaje conciernen a las relaciones directas de las empresas con los mayoristas y los minoristas bolivianos. Cuando hace años una corporación japonesa "descuidó" al mercado durante seis meses, todos sus banners y otra publicidad habían sido cambiadas por los de la competencia. Las transnacionales aprendieron que hay que estar con los comerciantes día a día y cara-a-cara para garantizar que se vendan sus productos y para acceder a información básica sobre el mercado.

El personal de las multinacionales está constantemente visitando los locales de venta para controlar la exhibición de los equipos, generar información para estudios de mercado y supervisar la llegada y el registro de los productos. Las relaciones de confianza entre los empleados de las marcas y los comerciantes son el requisito primordial para esta labor. Sobre

7 Sony Electronics ha sido la primera en abrir un "show room" en la calle Eloy Salmón en 2003. LG y Samsung Electronics siguieron su ejemplo en 2007 y 2012, respectivamente. Hoy en día se mantiene el local de Samsung Electronics, que ha invertido fuertemente en Bolivia en los últimos años. 
todo entre los comerciantes y vendedores más jóvenes y de la misma edad que los empleados de las transnacionales. Entre ellos se establecen fuertes lazos de amistad que difuminan la frontera entre el emprendimiento unipersonal y la empresa transnacional.

Otra manera de integrarse en el tejido sociocultural local es a través de las capacitaciones de las transnacionales, que han ido evolucionando desde seminarios técnicos hacia la modalidad de cena y fiesta. Asimismo, las empresas apoyan las fiestas de los comerciantes y hacen su acto de presencia en eventos importantes de las asociaciones gremiales.

\section{Conclusiones}

Estudiar el mercado de equipos electrónicos en Bolivia nos permite reenmarcar el estudio del comercio informal y popular. A lo largo de este texto se analizó un mercado que nace a partir del dinamismo del comercio popular, pero que se ha transformado -en un contexto donde el Estado no monopoliza la práctica regulatoria- en un mercado que incluye comercios unipersonales, micro-emprendimientos familiares, medianas y grandes empresas y empresas multinacionales. Los actores populares ylas transnacionales coexisten, se integran en relaciones de co-presencia y cooperan en las prácticas rutinarias de compra-venta, logística y marketing, pero se enfrentan en ocasiones puntuales, especialmente cuando se trata de asuntos de acceso al mercado y el control de la competencia.

En vez de estudiar las lógicas de ciertas economías como separadas (economía popular versus oficial, economía informal versus formal, economía ilegal versus legal), el presente estudio analiza las interacciones -tanto conflictivas como de cooperación- y los mimetismos entre agentes con diferentes posiciones, populares y transnacionales, con ideas y prácticas económicas distintas, las cuales, sin embargo, han co-construido un mercado lucrativo y extralegal.

Quisiera terminar proponiendo la noción de lo extralegal para estudiar y entender mejor el comercio en Bolivia. Lo extralegal hace referencia a

... prácticas que implican concepciones de derechos, moralidad y autoridad diferentes de lo que la ley formal y la burocracia establecen como legal. Prácticas extralegales implican la 
existencia de 'legalidades múltiples’ dentro y más allá de la división legal/ilegal definida por el Estado y el derecho internacional (Smart y Zerilli, 2014:226; traducción hecha por la autora) . $^{8}$

El mercado de artefactos electrónicos en La Paz y Bolivia es extralegal porque los arreglos básicos de cualquier mercado, regulación de la competencia, funcionan sin apelar al aparato estatal y al sistema jurídico. En este sentido, este mercado es extralegal porque las acciones colectivas para cuidar el mercado corresponden a normas y nociones sociales de lo legítimo. A pesar de que, desde el punto de vista jurídico, las acciones pueden ser interpretadas como conductas opuestas (unas anti-monopolizadoras, las otras, al contrario, anti-competitivas), desde el punto de vista de los comerciantes, sus acciones se justifican por combatir la competencia deslealy, por lo tanto, son para ellos legitimas. En este sentido competencia desleal es un término moral utilizado por los comerciantes para deslegitimar a los competidores. Aunque el origen del término se halla en el derecho comercial, ha sido re-semantizado acorde a nociones morales que predominan en este espacio de regulación no estatal.

Además, las prácticas de las empresas transnacionales también son extralegales porque establecen una forma de regulación de sus productos que busca aumentar sus ventas y beneficios y supervisar al mercado popular, pero no la legalidad estatal. Las transnacionales se nutren de las redes personalizadas predominantes en el mercado y utilizan incentivos monetarios para aumentar la venta de los productos originales con garantía. Al crear su propio espacio de regulación, las empresas transnacionales co-producen un mercado más allá de la ley y de las regulaciones oficiales, esto es, un mercado extralegal.

8 Extralegalidad también aplica a actividades no -o todavía no- definidas por la ley. Muchos ejemplos en la literatura vienen de las prácticas económicas en países de la ex Unión Soviética en transición de un sistema estatal-centralizado al capitalismo (Smart y Zerilli, 2014). 


\section{Referencias}

1. Altenburg, T. y J. Meyer-Stamer. 1999. "How to Promote Clusters? Policy Experiences from Latin America". World Development, 27(9),1693-1713. Disponible en: https:// doi. org/10.1016/S0305-750X(99)00081-9.

2. Aspers, P. y J. Beckert. 2008. Märkte. En: M. Maurer (ed.), Handbuch der Wirtschaftssoziologie, Wiesbaden. VS Verlag für Sozialwissenschaften.

3. Beckert, J. 2007. "The Great Transformation of Embeddedness. Karl Polanyi and the New Economic Sociology”. MPIfG Discussion Paper 07/1. Max Planck Institute for the Study of Societies.

4. Beckert, J. y M. Dewey. 2017. The Architecture of Illegal Markets. Towards an Economic Sociology of Illegality in the Economy. Oxford: Oxford University Press.

5. Beckert, J. y F. Wehinger. 2011. "In the Shadow Illegal Markets and Economic Sociology”, MPIfG Discussion Paper 11/ 9, Cologne. Max Planck Institute for the Study of Societies.

6. Blanes, J. 2017. El subsistema fronterizo boliviano en la globalización de los mercados ilegales. Quito: FLACSO.

7. Bourdieu, P. 2001. Las estructuras sociales de la economía. Buenos Aires: Manantial.

8. CEDLA. 1986. El sector informal en Bolivia. La Paz: CEDLA/FLACSO/ILDIS.

9. Clark, G. 1994. Onions Are My Husband: Survival and Accumulation by West African Market Women. Chicago: University of Chicago Press.

10. Dent, A. 2012. "Piracy, Circulatory Legitimacy, and Neoliberal Subjectivity in Brazil". Cultural Anthropology, 27(1): 28-49.

11. Dewey, M. 2016. "Porous Borders: The Study of Illegal Markets from a Sociological Perspective”. MPIfG Discussion Paper 16/2, Cologne. Max Planck Institute for the Study of Societies.

12. Dilley, R. 1992. "Contesting Markets. A general introduction to market ideology, imagery and discourse”. En: R. Dilley (ed.) Contesting Markets: Anthropology of Ideology, Discourse and Practice, Edinburgh: Edinburgh University Press.

13. Felzensztein, C. et al. 2014. "International Marketing Strategies in Industrial Clusters: Insights from the Southern Hemisphere". Journal of Business Research, 67 (5): 837-846. Disponible en: https://doi.org/10.1016/j.jbusres.2013.07.002. 
14. Fernández-Kelly, P. y J. Shefner. 2008. Out of the Shadows. Political Action and the Informal Economy in Latin America, University Park. The Pennsylvania State University Press.

15. Fligstein, N. 1996. "Markets as Politics: A political Cultural Approach to Market Institutions”. American Sociologial Review, 61 (4), 656-673.

16. Geertz, C. 1978. “The Bazaar Economy: Information and Search in Peasant Marketing”. The American Economic Review, 68(2), 28-32.

17. Giddens, A. 1990. The Consequences of Modernity. Stanford, CA. Stanford University Press.

18. Gray Molina, G. 2007. "El reto posneoliberal de Bolivia". Nueva Sociedad, № 209,118129.

19. Guyer,J.2004. Marginal gains: Monetary transactions in Atlantic Africa. Chicago. University of Chicago Press.

20. Giuliani, E., C. Pietrobelli y R. Rabellotti. 2005. "Upgrading in Global Value Chains: Lessons from Latin American Clusters”. World Development, 33 (4): 549-573.Disponible en: https://doi.org/10.1016/ j.worlddev.2005.01.002.

21. Lins Ribeiro, G. 2012. "La globalización popular y el sistema mundial no-hegemónico". Nueva Sociedad, 241: 36-62.

22. Mathews, G. y Y. Yang. 2012. "How Africans Pursue Low-End Globalization in Hong Kong and Mainland China”. Journal of Current Chinese Affairs, 41(2): 95-120.

23. Matos Mar, J. 1984. Desborde popular y crisis del Estado. Lima: Instituto de Estudios Peruanos.

24. Pereira, R. et al. (coord.) 2009. Para escuchar las voces de la calle: el comercio en vía pública en La Paz. La Paz: PIEB.

25. Pinheiro-Machado, R. 2013. "Copied products and the informal economy in Brazil and China. Outlining a comparison of development models". Vibrant, 9 (1):335-359.

26. Porter, M.E. 2000. "Location, Competition, and Economic Development: Local Clusters in a Global Economy”. Economic Development Quarterly, 14:15-34.Disponible en: https://doi.org/10.1177/089124240001400105.

27. Programa de Naciones Unidas para el Desarrollo (PNUD). 2005. Informe Temático de Desarrollo Humano en Bolivia: la economía boliviana más allá del gas. La Paz: Programa de Naciones Unidas para el Desarrollo. 
28. Portes, A. y R. Schauffler. 1993. "Competing Perspectives on the Latin American Informal Sector". Population and Development Review, 19(1), 33-60.

29. Quijano, A. 1998. La economía popular y sus caminos en América Latina. Lima: Mosca Azul Editores.

30. Rojas, B. 1992. La batalla en las calles. La participación de las organizaciones del comercio minorista en las reformas institucionales y politicas en la ciudad de La Paz. La Paz: OITCEDLA.

31. Rojas, R. 2016. La Feria 16 de Julio (jach 'a qhatu) de El Alto, Bolivia. ¿Territorio o aglomerado de exclusión? Quito: FLACSO.

32. Smart, A.y F.M. Zerilli. 2015. "Extralegality”. En: D. Nonini (ed.), A Companion to Urban Anthropology. West Sussex. John Wiley y Sons.

33. Tassi, N., A. Hinojosa y R. Canaviri (eds.) (2015). La economía popular en Bolivia: tres miradas. La Paz: Centro de Investigaciones Sociológicas/Vicepresidencia del Estado.

34. Tassi, N. et al. 2013. "Hacer plata sin plata". El desborde de los comerciantes populares en Bolivia. La Paz: Fundación PIEB.

35. Van Schendel, W. y I. Abraham. 2005. Illicit Flows and Criminal Things. States, Borders, and the Other Side of Globalization, Bloomington/Indianapolis. Indiana University Press.

36. Wanderley, F. 2012. "Pluralismo económico, mercado y Estado". En: G. Gosálvez (coord.), La Paz: Vicepresidencia del Estado Plurinacional de Bolivia/ Fundación Boliviana para la Democracia Multipartidaria.

37. White, H. 2002. Markets from Networks. Socioeconomic models of production. Princeton and Oxford, Princeton University Press.

38. Zelizer, V. 2011. Economic lives: how culture shapes the economy. Princeton, Princeton University Press. 